\title{
Status of the thermal model and chemical freeze-out
}

\author{
Jean Cleymans ${ }^{1, a}$ \\ ${ }^{1}$ UCT-CERN Research Centre and Department of Physics \\ University of Cape Town, Rondebosch 7701, South Africa
}

\begin{abstract}
A brief review is presented of the status of the thermal model and chemical freeze-out in relativistic heavy-ion collisions. Some interesting aspects at lower energies are emphasized.
\end{abstract}

Particle collisions at high energies produce large numbers of secondaries and it is natural to try a statistical-thermal model to analyse these. This type of analysis has a long and proud history [1-3]. In relativistic heavy ion collisions a new dimension is given to the model by the varying baryon density. The highly successful analysis of particle yields lead to the notion of chemical equilibrium which is by now a well-established tool in the analysis of relativistic heavy ion collisions, see e.g. [4]. Before the start of RHIC, only three points were present on the $T-\mu_{B}$ plane, showing a clear increase of the chemical freeze-out temperature, $T$, with increasing beam energy and an accompanying decrease of the baryon chemical potential $\mu_{B}[5,6]$. Substantial new knowledge became available in the following decade [7-9] and now cover almost the complete $T-\mu_{B}$ curve as shown in Fig. 1. A last substantial gap still exists in an energy region to be covered by the Beam Energy Scan at RHIC [10] and by FAIR and NICA. The results obtained at ALICE have been presented comprehensively recently in [11] with a chemical freeze-out temperature of $155 \pm 2 \mathrm{MeV}$ which is slightly lower than expected. A comparison [9] of three parameterizations is shown in Fig. 1. The resulting freeze-out curve in the $T-\mu_{B}$ plane can also be drawn in the energy density vs net baryon density plane as was first done in Ref. [12]. The resulting curve is shown in Fig. 2. This figure shows that the highest net baryon density will be reached in the beam energy covered by the RHIC/FAIR/NICA experiments. In view of the success of chemical freeze-out in relativistic heavy ion collisions, much effort has gone into finding models that lead to a final state in chemical equilibrium, see e.g. curve [13-16]. The corresponding dependence of the temperature and the chemical potential on beam energy is surprisingly smooth [9] as shown in Figs. 3 and 4. However, despite this smoothness in the thermal freeze-out parameters a roller-coaster is observed in several particle ratios, e.g. the horn in the $K^{+} / \pi^{+}$ratio and a similar strong variation in the $\Lambda / \pi$ ratio [17]. Again these strong variations are not observed in $p-p$ collisions. Within the framework of thermal-satistical models this variation has been connected to a change from a baryon domicated to a meson dominated hadron gas [14]. This conclusion is based on the observation that the entropy density divided by the temparature to the third power, $s / T^{3}$, is constant over the whole energy range. The change is shown in Fig. 5. Lines of constant value for the $K^{+} / \pi^{+}$ratio are shown in Fig. 6 where it can be seen that the absolute maximum in the thermal-statistical model hugs the chemical freeze-out line. The largest observed value is just barely compatible with this maximum. In the thermal-statistical model a rapid change is expected as the hadronic gas undergoes a transi-

\footnotetext{
ae-mail: jean.cleymans@uct.ac.za
} 


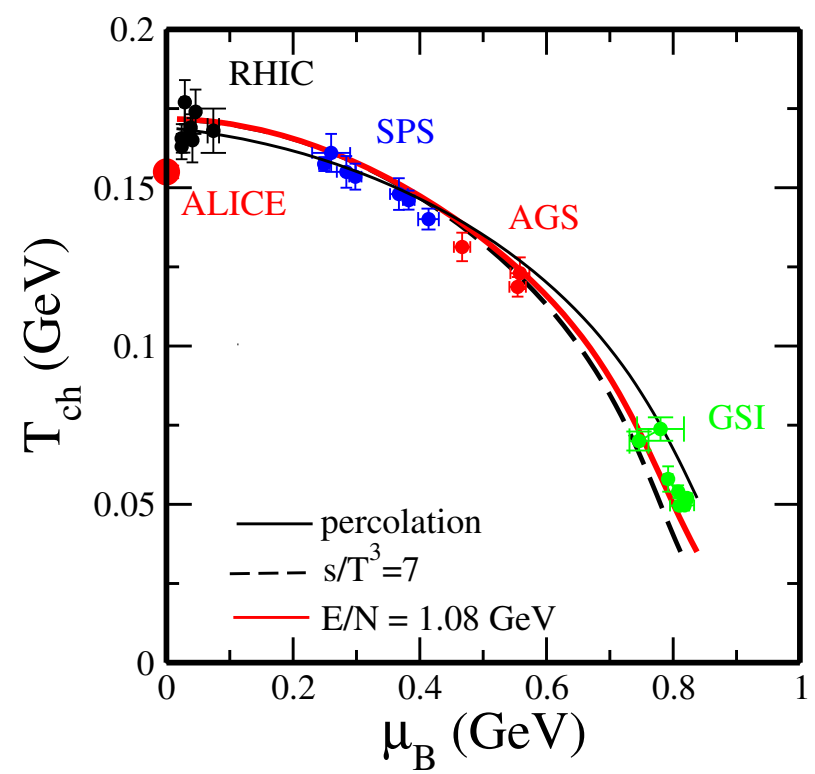

Figure 1. Chemical freeze-out temperature $T$ vs. the baryon chemical potential at different beam energies together with curves corresponding to various models [9].

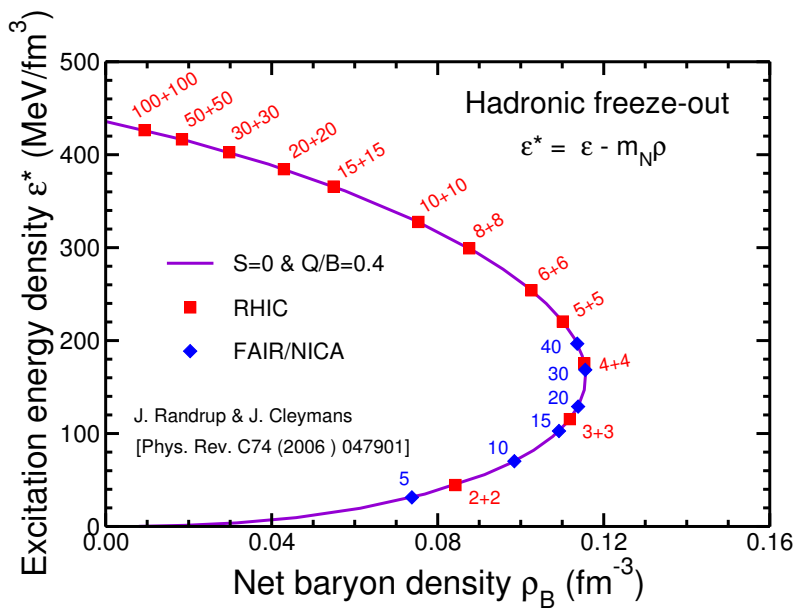

Figure 2. The hadronic freeze-out line in the $\rho_{B}-\epsilon$ phase plane as obtained from the values of $\mu_{B}$ and $T$ that have been extracted from the experimental data in [9]. The calculation employs values of $\mu_{Q}$ and $\mu_{S}$ that ensure $\langle S\rangle=0$ and $\langle Q\rangle=0.4\langle B\rangle$ for each value of $\mu_{B}$. Also indicated are the beam energies (in $\mathrm{GeV} / \mathrm{N}$ ) for which the particular freeze-out conditions are expected at either RHIC or FAIR or NICA.

tion from a baryon-dominated to a meson-dominated gas. The transition occurs at a temperature $T$ $=151 \mathrm{MeV}$ and baryon chemical potential $\mu_{B}=327 \mathrm{MeV}$ corresponding to an incident energy of $\sqrt{s_{N N}}=11 \mathrm{GeV}$. Thus the strong variation seen in the particle ratios corresponds to a transition from a baryon-dominated to a meson-dominated hadronic gas. This transition occurs at a 
ICNFP 2014

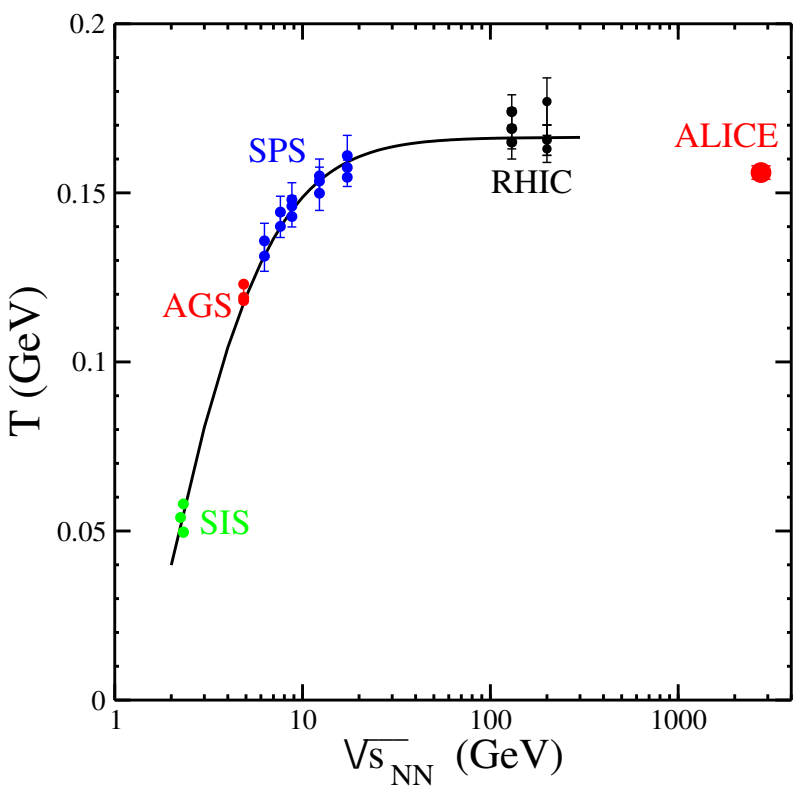

Figure 3. Chemical freeze-out temperature $T$ as a function of the beam energy.

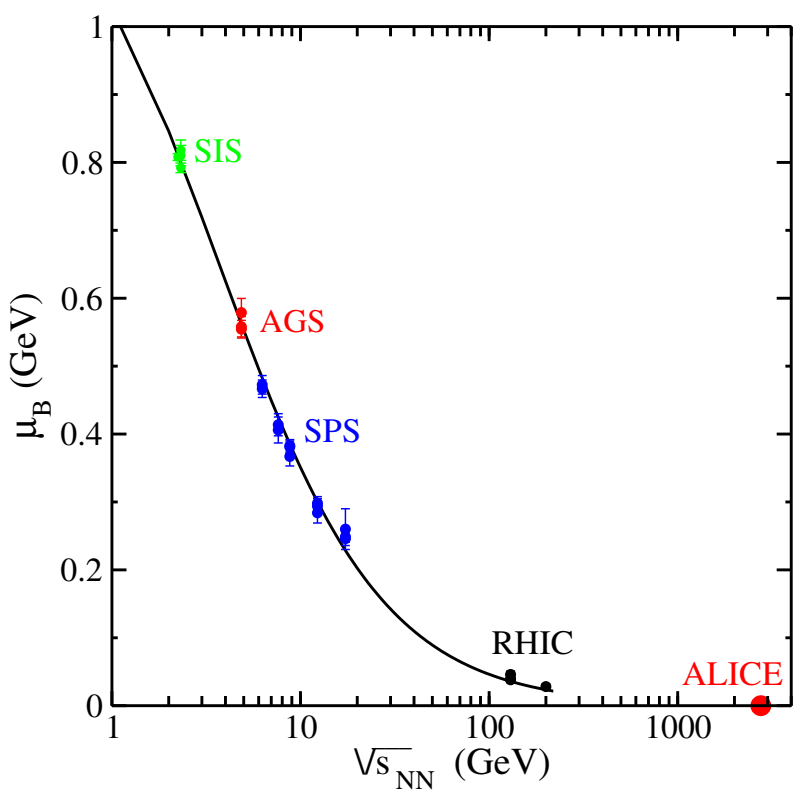

Figure 4. Chemical freeze-out baryon chemical potential $\mu_{B}$ as a function of the beam energy. 


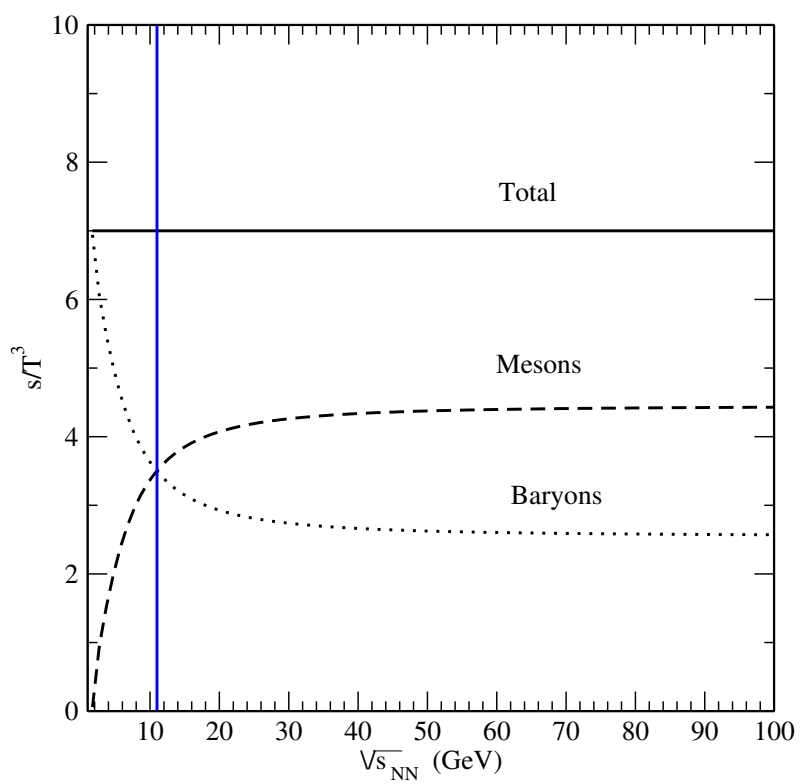

Figure 5. The $s / T^{3}$ ratio calculated in the thermal-statistical model along the constant value consistent with chemical freeze-out. Also shown are the contributions from the mesons and the baryons.

- temperature $T=151 \mathrm{MeV}$,

- baryon chemical potential $\mu_{B}=327 \mathrm{MeV}$,

- energy $\sqrt{s_{N N}}=11 \mathrm{GeV}$.

In the statistical model this transition leads to peaks in the $\Lambda /\langle\pi\rangle, K^{+} / \pi^{+}, \Xi^{-} / \pi^{+}$and $\Omega^{-} / \pi^{+}$ratios. However, the observed ratios are sharper than the ones calculated in thermal-statistical models.

We present a review of data obtained in relativistic heavy ion collisions and show that there is a gap around $11 \mathrm{GeV}$ where more and better precise measurements are needed. The theoretical interpretation can only be clarified by covering this energy region. In particular the strangeness content needs to be determined, data covering the full phase space $(4 \pi)$ would be very helpful to determine the thermal parameters of a possible phase transition and the existence of a quarkyonic phase as has been discussed recently [18].

\section{Acknowledgments}

The numerous contributions by H Oeschler, J. Randrup, K. Redlich, E. Suhonen and S. Wheaton are gratefully acknowledged.

\section{References}

[1] H. Koppe, Z. Naturforschung, 3a, 251, 1948.

[2] E. Fermi, Progr. Theor. Phys, (Kyoto) 5, 570, 1950.

[3] R. Hagedorn, Nuovo Cimento Suppl. 3, 147, 1965.

[4] J. Cleymans and H. Satz, Zeitschrift für Physik C57, 135, 1993. 


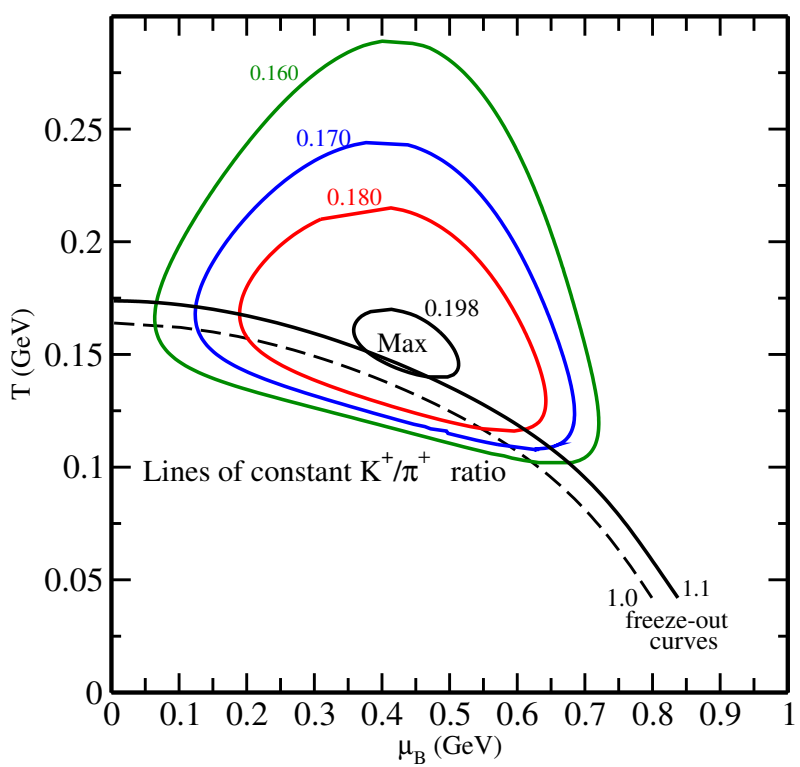

Figure 6. Lines of constant value of the $K^{+} / \pi^{+}$ratio in the $T-\mu_{B}$ plane showing a clear maximum in this ratio close to the boundary given by the chemical freeze-out line.

[5] F. Becattini, J. Cleymans, A. Keränen, E. Suhonen and K. Redlich, Physical Review C64, 024901, 2001.

[6] P. Braun-Munzinger, K. Redlich, J. Stachel, published in Hwa, R.C. (ed.) et al.: Quark-Gluon Plasma 491-599, 2003.

[7] A. Andronic, P. Braun-Munzinger, J. Stachel, Nucl. Phys. A772, 167, 2006.

[8] J. Manninen, F. Becattini, M, Gazdzicki, Phys. Rev. C73, 044905, 2006.

[9] J. Cleymans, K. Redlich, H. Oeschler, S. Wheaton, Phys. Rev. C73, 034905 (2006).

[10] Shusu Shi (for the STAR Collaboration), these proceedings.

[11] M. Floris, arXiv:1408.6403v3 [nucl-ex]

[12] J. Randrup, J. Cleymans, Phys. Rev. C74, 047901, 2006.

[13] V. Magas, H. Satz, Eur. Phys. J. C32, 115, 2003.

[14] J.Cleymans, H. Oeschler, K. Redlich, S. Wheaton, Phys. Lett. B615, 50, 2005.

[15] T.S. Biro and J. Cleymans, Phys. Rev. C78, 034902, 2008.

[16] P. Castorina, A. Iorio, H. Satz, arXiv:1409.3104 [hep-ph]

[17] C. Alt et al. (NA49 collaboration), Physical Review C77, 024903, 2008.

[18] A. Andronic et al., Nucl. Phys. A837, 65, 2010. 